\title{
Evaluation of Effectiveness of Anatomical Rotation Change Image by Aid Tool in Shoulder MRArthrography
}

\author{
Hyeonggyun Kim*, Jaeeun Jung**, Hongmoon Jung** \\ Department of Intelligent Mechanical Engineering, Graduate school of Kumoh of National institute of technology, \\ Department of Radiological Science, Daegu Health College**
}

\section{Shoulder MRArthography 검사 시 보조기구를 이용한 해부학적 회전 변화 영상에 대한 유용성 평가}

\author{
김형균 ${ }^{*}$, 정재은**, 정홍문** \\ 금오공과대학교 대학원 지능기계공학과*, 대구보건대학교 방사선과**
}

\begin{abstract}
Shoulder MRArthrography was performed to get an accurate diagnosis about complex anatomical structure in shoulder joint. We carried out how the changes of anatomical rotations in shoulder joint could bring certain diagnosis effects on MRI images for various shoulder humerus positions; Neutral position, Internal rotation position and External rotation position. In addition, we prepared an aid tool in oder to maintain the right posture of a patient. This aid tool was made by adapting Modeling Design Program. By virtue of this aid, we obtained the following result. Shoulder MR Arthrography by the External rotation position for anatomical structure diagnosis was the most suitable in diagnostic evaluations of important anatomical structures in shoulder joint such as Biceps tendon, Supera-spiatus tendon, Sub-scapularis tendon, Labrum and Sub-acromial space.
\end{abstract}

Key Words : MRArthrography, Shoulder joint MRI, MRArthrography aid tool

\section{요야}

자기공명 관절 조영술(Shoulder MR Arthrography)는 견관절(Shoulder joint) 의 복잡한 해부학적 구조에 관하여 정확한 영상평가를 진단하기 위해 시행 한다. 우리는 어께 상완골(Shoulder humerus) 의 다양한 position인 Neutral position, Internal rotation position, External rotation position 에 관하여 Shoulder joint의 해부학 적인 회전 변화가 견관절 자기공명(Shoulder MRI) 영상에 어떤 진단적 결과를 가져오는가에 대해 상호 비교 하였다. 또한 환자의 정확 한 촬영자세 유지를 위해 촬영 보조기구를 만들었다. 이 보조기구는 Modeling 설계에 의해 우리가 직접 제작한 자기공 명 견관절 조영술 보조 기구이다. 이 보조기구를 사용하여 촬영한 결과 다음과 같은 결론을 도출해 내었다. External rotation position 에 의한 Shoulder MRArthrography 검사가 Shoulder joint의 중요 해부학적 구조인 Biceps tendon, Supera-spiatus tendon, Sub-scapularis tendon, Labrum, Sub-acromial space 의 해부학적 평가에서 제일 적합하다 는 진단적 평가를 얻었다.

중심단어: 자기공명관절조영술, 견관절자기공명, 자기공명견관절조영술 보조기구 


\section{I. 서론}

$\mathrm{MRI}$ 의 도입은 방사선과 진단영역의 큰 변화를 가 져 다 주었다. 또한 높은 해상도의 이미지로 검사의 정확성을 높였다. 이후 hardware와 software의 발전으로 검사시간의 단축과 화질 향상이 꾸준히 진행되었다. 검사자인 방사선사들도 검사에 필요한 기술을 개발함 으로서 진단의 정확성을 위하여 노력하고 있다. 어깨 관절(Shoulder joint)[Fig. 1-1]는 다른 관절과 달리 해부 학적으로 humerus와 scapular가 맞 닫는 부분(Glenoid joint)이 다른 관절에 비하여 적고 그에 따라 인대가 많 이 존재하여 회전력이 크다는 장점 있는 반면, 사고나 질환에 노출되는 확률 또한 크다는 단점이 있다. 이러 한 견관절 질환 ${ }^{[1]}$ 의 발생과 복잡한 해부학적 구조에 대한 진단학적 영상평가를 위해 Shoulder MRArthro graphy 검사 ${ }^{[2],[3],[9],[11]}$ 시 자체 제작한 보조기구를 사용 하여 상완골의 해부학적 회전 ${ }^{[4][5]}$ 에 따른 변화가 $\mathrm{MRI}^{[11],[12],[13]}$ 영상에 어떤 진단적 유용성을 주는지 상 호 비교 하고자 하였다.

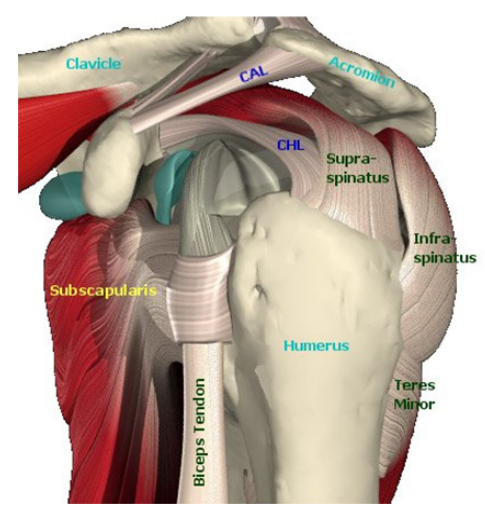

Fig. 1-1Shoulder joint ${ }^{[14]}$ 견관절에 존재하는 다양한 관절과 해부학적인 구조물을 보여주고 있다.연부조직의 다양성으로 인해 MRArthrography를 시행하게 된다.

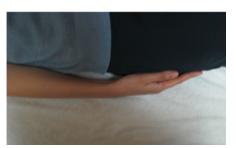

Neutral

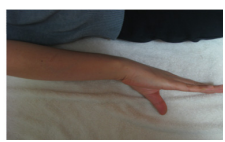

Internal $\mathrm{R}$.

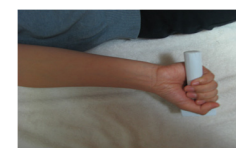

External R.
Fig. 1-2 Patient position
견관절에 존재하는 근육및 해부학구조를 보기위한 다양한 포지션방법으로 EXternal 자세는 계속적으로 유지하기 어려 움으로 그림과같이 고정보조기구를 이용하여 촬영하였다.

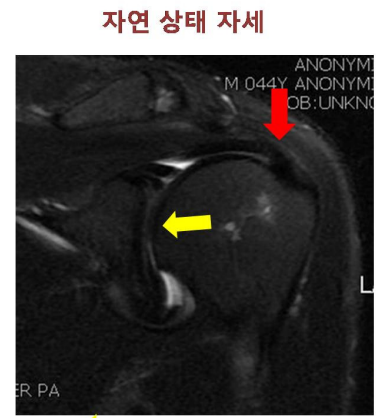

Shoulder joint
보조 기구 외전

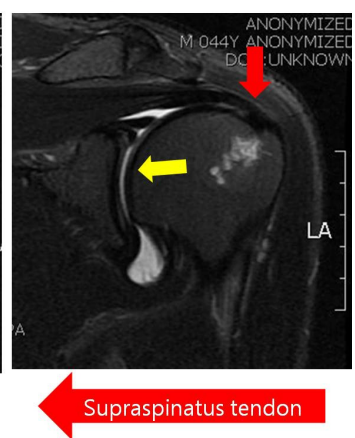

Fig. 1-3 Shoulder joint Obl-Coronal T2WI fat-suppression

기구를 사용하지 않았을때 MR영상 (왼쪽)과 본실험에서 고안 한 기구를 사용했을때의 MR영상(오른쪽)비교로서 기구를 사 용했을시에 Supraspinatus tendon과 같은 다양한 해부학적 구 조물을 보다 정확히 관찰할수 있게 해준다.

\section{II. 실험 및 방법}

\section{1. 실험장비 및 기구}

1) 실험장비 : GE社의 $1.5 \mathrm{~T}$ MRI Signa HDXT

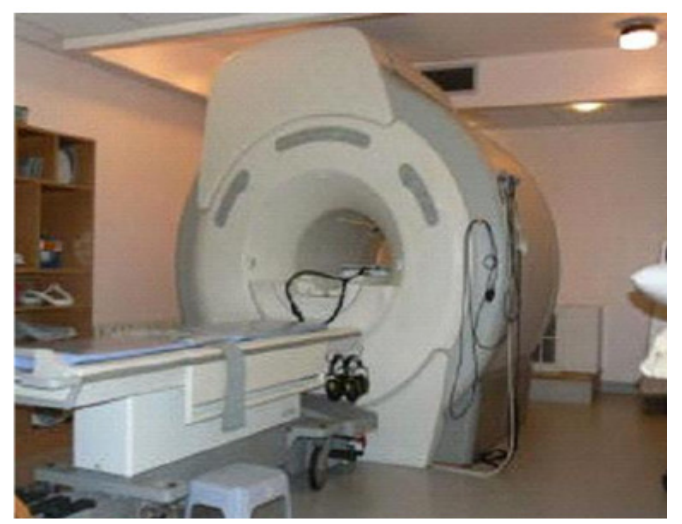

Fig. 2 GE社의 1.5T MRI Signa HDXT 
2) 실험기구 : Modeling 설계로 직접 나무제작
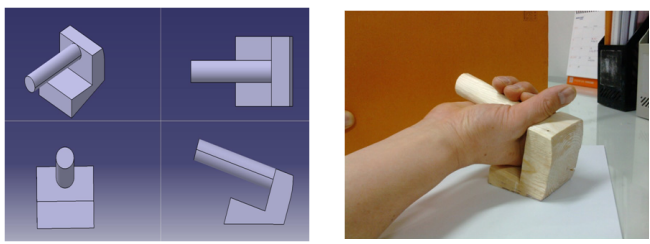

Fig. 3 aid tool Modeling

상완골과 어깨 관절을 최대로 External position 자세 를 취한 후 그 상태에서 지지체 나무 부목을 잡게 하 여 지지체와 부목의 각도는 약 30 도 정도로 유지하여 $3 \mathrm{D}$ 프로그램 Cartia를 사용하여 3차원영상을 먼저 만 든 후 나무로 보조기구를 제작하였다.

\section{2. 실험방법}

1) 2011년 1월부터 3월까지 본원을 방문하여 Shoulder MRArthrography 검사를 시행한 32 례중 18 례 의 영상을 비교 분석한 결과를 바탕으로 하였다.

2) [Fig. 3]과 같이 환자는 보조기구를 잡고 Neutral position, Internal rotation position, External rotation position의 자세로 Oblique Coronal T2WI Fat Saturation 검사를 시행하였다.

3) 평가대상 해부학적 구조는 Biceps tendon, Supera-spiatus tendon, Sub-scapularis tendon, Labrum, Sub-acromial space 이며 평가표[Table 1 ]를 만들어 골 관절 영상의학과 전문의 1 명, 견관절 정형외과 전문의 1 명, MRI 국제 전문방사선사 2명이 항목별로 매우용 이 3점, 평가용이 2점, 평가어려움 1점을 기준으로 평 가하여 얻은 점수를 분석하였다.

\section{III. 결과}

Modeling 설계에 의해 자체 제작한 보조기구를 사용 하여 검사를 시행하였으며 어깨관절의 회전에 따른 보조기구의 유용성에 대한 결과를 전문의와 방사선사 를 통하여 Table 2와 같이 얻었다. 총 32례 중 18 례에 대해 견관절 5 가지 주요 부위에 대해 3 가지 각 자세별 로 매우용이 3점, 평가용이 2점, 평가어려움 1점으로 평가하여 실제 점수화 된 18 례의 합계 점수이며 백분
율은 최고 받을수 있는 점수 216(매우용이 3점 * 평가 자 4명 * 18 례 )점을 $100 \%$ 로 했을때 상대 $\%$ 를 나타낸 것이다.

Table 1. evaluation chart

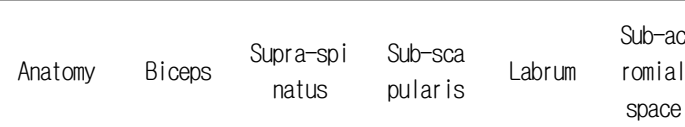

\begin{tabular}{|c|c|c|c|c|c|c|}
\hline \multirow{9}{*}{$\begin{array}{l}P \\
0 \\
\text { S } \\
i \\
\vdots \\
i \\
0 \\
n\end{array}$} & \multirow{3}{*}{ 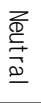 } & 3 & 3 & 3 & 3 & 3 \\
\hline & & 2 & 2 & 2 & 2 & 2 \\
\hline & & 1 & 1 & 1 & 1 & 1 \\
\hline & \multirow{3}{*}{$\begin{array}{l}\stackrel{\bar{\Xi}}{\underline{\underline{D}}} \\
\underline{\bar{\Xi}}\end{array}$} & 3 & 3 & 3 & 3 & 3 \\
\hline & & 2 & 2 & 2 & 2 & 2 \\
\hline & & 1 & 1 & 1 & 1 & 1 \\
\hline & \multirow{3}{*}{ 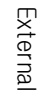 } & 3 & 3 & 3 & 3 & 3 \\
\hline & & 2 & 2 & 2 & 2 & 2 \\
\hline & & 1 & 1 & 1 & 1 & 1 \\
\hline
\end{tabular}

Table 2. Total scores ( $\max : 216=3 * 4$ person $* 18$ )

\begin{tabular}{|c|c|c|c|}
\hline Division & Neutral & External & Internal \\
\hline Biceps & $179(82.87 \%)$ & $191(88.43 \%)$ & $147(68.06 \%)$ \\
\hline $\begin{array}{c}\text { Supra- } \\
\text { spinatus }\end{array}$ & $176(81.48 \%)$ & 199(92.13\%) & $165(76.39 \%)$ \\
\hline $\begin{array}{c}\text { Sub- } \\
\text { scapular is }\end{array}$ & $184(85.18 \%)$ & $188(87.04 \%)$ & $159(73.61 \%)$ \\
\hline Labrum & $197(91.20 \%)$ & $199(92.13 \%)$ & $195(92.13 \%)$ \\
\hline $\begin{array}{c}\text { Subacromial } \\
\text { space }\end{array}$ & $195(90.28 \%)$ & $195(90.28 \%)$ & $195(92.13 \%)$ \\
\hline $\begin{array}{c}\text { Total } \\
\text { (max: } 1080 \\
\text { Point) }\end{array}$ & $941(87.13 \%)$ & $972(90 \%)$ & $861(79.72 \%)$ \\
\hline
\end{tabular}

Table 3 은 18 례 중 견관절 주요 부위에 대해 자세별 매우용이 3점을 받은 건수로 평가자 4명이 합계 한 것 이다. 백분율은 최고 받을수 있는 72 건에 대한 상대비 율로 백분율이 높을수록 우수한 진단적 영상이다.

Table 3. very good (3 point)

\begin{tabular}{cccc}
\hline Division & Neutral & External & Internal \\
\hline Biceps & $48(66.67 \%)$ & $50(69.44 \%)$ & $20(27.78 \%)$ \\
\hline $\begin{array}{c}\text { Supra- } \\
\text { spinatus }\end{array}$ & $48(66.67 \%)$ & $56(77.78 \%)$ & $29(40.28 \%)$ \\
\hline $\begin{array}{c}\text { Sub- } \\
\text { scapular is }\end{array}$ & $46(63.89 \%)$ & $48(66.67 \%)$ & $23(31.94 \%)$ \\
\hline $\begin{array}{c}\text { Labrum } \\
\text { Subacromial } \\
\text { space }\end{array}$ & $54(75 \%)$ & $56(77.78 \%)$ & $48(66.67 \%)$ \\
\hline $\begin{array}{c}\text { Total } \\
\text { (max: } 1080 \\
\text { Point })\end{array}$ & $248(68.89 \%)$ & $262(72.78 \%)$ & $170(47.22 \%)$ \\
\hline
\end{tabular}


Table 4는 18 례 중 견관절 주요 부위에 대해 자세별 평가용이 2점을 받은 건수를 말하며 평가자 4명이 합 계 한 것으로 백분율은 최고 받을수 있는 72 건에 대한 상대비율이다. 백분율이 높을수록 용이한 진단적 영상 이라 할 수 있다.

\begin{tabular}{cccc}
\multicolumn{4}{l}{ Table 4. good (2 point)) } \\
\hline Division & Neutral & External & Internal \\
\hline Biceps & $26(36.11 \%)$ & $20(27.78 \%)$ & $38(52.78 \%)$ \\
\hline $\begin{array}{c}\text { Supra- } \\
\text { spinatus }\end{array}$ & $28(38.89 \%)$ & $13(18.06 \%)$ & $36(50 \%)$ \\
\hline $\begin{array}{c}\text { Sub- } \\
\text { scapular is }\end{array}$ & $23(31.94 \%)$ & $20(27.78 \%)$ & $41(56.94 \%)$ \\
\hline $\begin{array}{c}\text { Labrum } \\
\text { Subacromial } \\
\text { space }\end{array}$ & $17(23.61 \%)$ & $15(20.83 \%)$ & $22(30.56 \%)$ \\
\hline $\begin{array}{c}\text { Total } \\
\text { (max: } 1080 \\
\text { Point) }\end{array}$ & $112(31.11 \%)$ & $18(25 \%)$ & $21(29.17 \%)$ \\
\hline
\end{tabular}

Table 5 는 18 례 중 견관절 주요 부위에 대해 자세별 평가어려움 1 점을 받은 건수를 말하며 평가자 4명이 합계한 것으로 백분율은 최고 받을수 있는 72 건에 대 한 상대비율이며 백분율이 높을수록 진단적 가치가 낮은 영상이라 할 수 있다.

Table 5. Poor(1point) (max:72 cases, min:0 case)

\begin{tabular}{cccc}
\hline 구분 & Neutral & External & Internal \\
\hline Biceps & $2(2.78 \%)$ & $1(1.39 \%)$ & $12(16.67 \%)$ \\
\hline $\begin{array}{c}\text { Supra- } \\
\text { spiatus }\end{array}$ & $0(0 \%)$ & $0(0 \%)$ & $6(8.33 \%)$ \\
\hline $\begin{array}{c}\text { Sub- } \\
\text { scapular is }\end{array}$ & $2(2.78 \%)$ & $3(4.17 \%)$ & $6(8.33 \%)$ \\
\hline \begin{tabular}{c} 
Labrum \\
\hline $\begin{array}{c}\text { Subacromial } \\
\text { space }\end{array}$
\end{tabular} & $1(1.39 \%)$ & $1(1.39 \%)$ & $1(1.39 \%)$ \\
\hline $\begin{array}{c}\text { Total } \\
\text { (max: } 1080 \\
\text { Point })\end{array}$ & $6(1.67 \%)$ & $1(1.39 \%)$ & $0(0 \%)$ \\
\hline
\end{tabular}

\section{IV. 고찰 및 결론}

Shoulder MRArthro 검사의 실험 결과 해부학적 회전 변화는 External Rotation Position ${ }^{[6]}$ 이 가장 유용한 영 상 평가임을 알 수 있었다. 또한 해부학적 영역별 평 가 $^{[7]}$ 에 있어서 Labrum, Subacromial Space는 해부학적
회전 여부와 관계없이 평가가 유용 하였고, Biceps, Supraspinatus, Subscapularis 의 tendon은 External, Neutral, Internal 순으로 평가가 유용 하였다. 일반적인 기구를 사용하지 않은 상태에서의 Shoulder MRArthro 검사는 정확한 견관절의 다양한 해부학적 위치정보를 정확히 확인 할 수 없다. 따라서 이것을 극복하고자 우리가 만든 보조기구를 사용하면 자연스레 External Rotation Position 이 유지가 되어 환자가 고의로 힘을 주지 않아도 자세가 자연스레 유지된다. 또한 보조기 구를 사용하여 검사를 함으로서 환자의 심리적 안정 과 해부학적 회전 변화를 볼 수 있었다. 또한 이 기구 를 사용하지 않는다면 환자가 인위적으로 External Rotation Position 을 유지해야한다. 결국 이것은 영상의 움직임 등으로 인한 화질 저하를 가져다 주는 결과를 종종 볼수 있었다. 그러나 본 연구를 통하여 얻은 Shoulder MRArthro 검사 보조기구를 사용한다면 이러 한 움직임에 의한 보정도 가져다주어 영상판단에 있 어 정확한 해부학적 진단이 높다고 사료된다. 본 연구 에 있어서 앞으로 좀 더 연구해야 할 점은 보조기구가 다양한 회전각도를 만들 수 있는 정밀한 보조기구가 개발 된다면 그 유용성은 매우 높으리라고 생각한다. 본 연구는 Shoulder MRArthro 검사 환자에게 설명과 보조기구를 사용 했을때의 차이를 충분히 설명하여 보다 진단학적으로 유용 할 수 있다는 동의를 얻어 촬 영 후 통계를 내었다. 결론적으로 해부학적으로 견관 절은 다양한 근육이 존재한다. 때문에 자유로운 반경 과 다양한 회전을 할수 있다. 견관절은 움직임이 다양 한 반면, 이로인하여 다양한 근육손상을 가져다 준다. 때문에 Shoulder MRArthro 검사시에 우리가 제작한 보 조기구 사용은 보다 정확한 견관절의 다양한 구조물 의 해부학적 이미지를 제공 할 수 있다.

\section{참고문헌}

[1] Saleem AM, Lee JK, Novak LM.AJR. Usefulness of the abduction and external rotation views in shoulder MR arthrography. Am J Roentgenol, Vol. 191, No. 4, pp.1024-30, 2008.

[2] Beltran J, Rosenberg ZS, Chandnani VP, Cuomo F, Beltran S, Rokito A. Glenohumeral instability : evaluation with MR arthrography. Radiographics, Vol. 17,pp.657-673,1997.

[3] Stoller DW. MR arthrography of the glenohumeral joint. Radiol. 
Clin North Am, Vol .35, pp97-116,1997.

[4] Andrews JR, Carson WG Jr, McLeod WD. Glenoid labrum tears related to the long head of the biceps. Am J Sports Med, Vol. 13, pp.337-341,1997.

[5] Pappas AM, Goss TP, Kleinman PK. Symptomatic shoulder instability due to lesions of the glenoid labrum. Am J Sports Med, Vol. 11, pp279-288,1997.

[6] Herold T, Bachthaler M, Hamer OW, Hente R, Feuerbach S, et al..Indirect MR arthrography of the shoulder: use of abduction and external rotation to detect full-and partial-thickness tears of the supraspinatus tendon.. Radiology, Vol. 240, No. 1, pp.152-60, 2006.

[7] Chandnani VP, Gagliardi JA, Murnane TG, et al. Glenohumeral ligaments and shoulder capsular mechanism: evaluation with MR arthrography. Radiology, Vol. 196, pp.27-32, 1997.

[8] Gross ML, Seeger LL, Smith JB, Mandelbaum BR, Finerman GA.Magnetic resonance imaging of the glenoid labrum. Am J Sports Med, Vol. .18, pp.229-234, 1997.

[9] Wischer TK, Bredella MA, Genant HK, Stoller DW, Bost FW, Tirman PF.Perthes lesion (a variant of the Bankart lesion): MR imaging and MR arthrographic findings with surgical correlation. AJR Am J Roentgenol, J, Vol. 178, No. 1, pp.233-7, 2002

[10] Choi JA, Suh SI, Kim BH, Cha SH, Kim MG, Lee KY, Lee CH.Comparison between conventional MR arthrography and abduction and external rotation MR arthrography in revealing tears of the antero-inferior glenoid labrum. Korean J Radiol, Vol. 2, No. 4, pp.216-21, 2001.

[11] Kwak SM, Brown RR, Trudell D, Resnick DGlenohumeral joint: comparison of shoulder positions at MR arthrography. .Radiology, Vol. 208, No. 2, pp.375-80, 1998.

[12] Cvitanic O, Tirman PF, Feller JF, Bost FW, Minter J, Carroll KW.Using abduction and external rotation of the shoulder to increase the sensitivity of MR arthrography in revealing tears of the anterior glenoid labrum. AJR Am J Roentgenol, Vol. 169, No. 3, pp.837-44, 1997.

[13] Flannigan B, Kursunoglu-Brahme S, Snyder S, Karzel R, Del Pizzo W, Resnick D. MR arthrography of the shoulder: comparison with conventional MR imaging. AJR Am J Roentgenol, Vol. 155, pp.829-832, 1997.

[14] http://www.drsarahsimison.com(rotator-cuff-muscles-and-chiropracti c-care-part-1) 